\title{
Diabetes Increases the Incidence of Levodopa-Induced Dyskinesia in Parkinson's Disease; A Case-Control Study
}

\author{
Marzieh Mirzae 1(D), Mohammad Hossein Abbasi 1(D), Gholam Ali Shahidi 1 (1) \\ Qodsiye Hosseinifakhr ${ }^{2}$ (D), Farzad Sina ${ }^{1}$ (D), Seyed Amirhassan Habibi ${ }^{1}$ (i)
}

1. Dept. of Neurology, Rasoul Akram Hospital, Iran University of Medical Science, Tehran, Iran

2. Dept. of Pharmacology and Toxicology, Faculty of Pharmacy, Tehran University of Medical Science, Tehran, Iran

\begin{tabular}{|c|c|}
\hline Article Info & ABSTRACT \\
\hline dol $10.30699 /$ jambs.29.136.257 & \multirow{2}{*}{$\begin{array}{l}\text { Background \& Objective: Dyskinesia is a debilitating complication of } \\
\text { Parkinson's disease (PD), which appears due to some known risk factors. The effect } \\
\text { of diabetes and high plasma glucose on the manifestation of dyskinesia has been } \\
\text { evaluated in just a few previous reports. The current study aimed to assess the } \\
\text { mentioned correlation. }\end{array}$} \\
\hline $\begin{array}{l}\text { Received: 2020/08/10; } \\
\text { Accepted: 2021/03/14; } \\
\text { Published Online: } 04 \text { Apr 2021; }\end{array}$ & \\
\hline \multirow{2}{*}{$\begin{array}{l}\text { Use your device to scan and read the } \\
\text { article online }\end{array}$} & $\begin{array}{l}\text { Materials \& Methods: In this case-control study, } 88 \text { patients with PD were enrolled } \\
\text { and categorized into two equal groups of diabetic and non-diabetic patients. They were } \\
\text { selected from the movement disorder clinic in Rasoul Akram Hospital, Tehran, Iran. } \\
\text { Patients were evaluated regarding the presence of dyskinesia and its characteristics, } \\
\text { besides the assessment of other clinical parameters. }\end{array}$ \\
\hline & $\begin{array}{l}\text { Results: The prevalence of dyskinesia in diabetics, compared to non-diabetics, } \\
\text { showed a higher rate }(P=0.033) \text {. Baseline parameter equality was confirmed to } \\
\text { exclude the confounding bias effect. Simultaneous involvement of upper and lower } \\
\text { extremities (right after drug intake) was the most prevalent sign of dyskensia in } \\
\text { diabetic patients with PD. }\end{array}$ \\
\hline \multirow{2}{*}{$\begin{array}{l}\text { Corresponding Information: } \\
\text { Seyed Amirhassan Habibi, } \\
\text { Dept. of Neurology, Rasoul Akram } \\
\text { Hospital, Iran University of Medical } \\
\text { Science, Tehran, Iran } \\
\text { E-Mail: Habibi.sah@iums.ac.ir } \\
\text { habibisaiyedamir@yahoo.com }\end{array}$} & $\begin{array}{l}\text { Conclusion: The comorbidity of PD and diabetes showed a higher prevalence of } \\
\text { levodopa-induced dyskinesia (LID) in PD; this result was obtained based on } \\
\text { homogeneity of the two groups in manners of age, disease, treatment duration and } \\
\text { the dosage of levodopa. }\end{array}$ \\
\hline & $\begin{array}{l}\text { Keywords: Diabetes mellitus, Dyskinesia, Levodopa-induced dyskinesia, } \\
\text { Parkinson's disease }\end{array}$ \\
\hline (c) (1) (8) & $\begin{array}{l}\text { ns of the Creative Commons } \\
\text { citation. }\end{array}$ \\
\hline
\end{tabular}

\section{Introduction}

Parkinson's disease (PD) is a systemic neurodegenerative disorder, affecting dopaminergic interactions inside the basal ganglia; finally, it results in an intermittent transfer of unwanted stimulatory signals into the corticospinal tract and disease manifestations. The corticospinal tract is the final affected portion of dopaminergic neurons through inhibition or stimulation of thalamic stimulatory signals to the motor cortex as a result of basal ganglia neuronal interactions (1-3).

The results of 5 cohort studies (in the United States) have been merged with information from the Medicare program until 2010; a prevalence of 572 patients in 100000 individuals (over 45 years old) was obtained. It is estimated to grow up to 1238000 patients in 2030 (4).

Patients with a history of head trauma, inflammatory bowel disease (IBD), and diabetes mellitus (DM) are significantly at a higher risk for PD involvement, while genetic factors have a minor role in it $(5,6,7)$.
In the past 56 years, levodopa has been introduced and used in clinical settings. Inhibitors of peripheral degrading enzymes of levodopa were added to the treatment regimen to increase drug efficacy and decrease peripheral side effects $(8,9)$.

The prevalence of dyskinesia in patients with PD is estimated to be in a wide range of $9 \%-80 \%$. Prolonged consumption of levodopa is the main known reported risk factor for the incidence of dyskinesia. Furthermore, the younger-onset age of PD is the other known risk factor (8).

Hyperactivation of type 1 dopamine receptors is introduced as a signaling pathway, which plays a vital role in levodopa-induced dyskinesia (LID) (10). Hyperglycemic state induces hypersensitization to dopamine receptors, which leads to chronic stimulation, as levodopa consumption leads to the subsequent occurrence of dyskinesia (11). 
DM has been shown to be correlated with the increased incidence of PD by $40 \%$ compared to the normal population rate $(6,12,13)$. The prognosis of $\mathrm{PD}$ is worsened by the simultaneous presence of DM in different clinical aspects, including the postural and motor signs (14). Diabetes comorbidity in patients with PD is responsible for $36 \%$ of aggravation in their clinical status (15).

The presence of dyskinesia and other motor signs in patients with PD have been evaluated by a few previous reports; they are more prevalent in patients with diabetes compared with non-diabetic patients with $\operatorname{PD}(14,16)$.

$\mathrm{DM}$ is a chronic propagating disease, estimated to involve 350 million people worldwide until 2025. Comorbidity between diabetes and PD has been reported in numerous previous studies. This co-occurrence is suggested to be due to the factors such as common causal genes, common disturbances in protein synthesis, or mitochondrial disorders, which result in the simultaneous occurrence of both diseases (13).

PD imposes a high psychosocial and economic burden attributable to motor disturbances; dyskinesia is the most prominent side effect, which affects health-related quality of life (HRQOL) in patients with PD (11). This study aimed to find a correlation between the incidence of dyskinesia and the comorbidity of PD and DM.

\section{Materials and Methods}

In this case-control study, the risk of simultaneous involvement of PD and DM was assessed in the presence of dyskinesia. Among those who referred to the Neurology Clinic of Rasoul Akram Hospital, 88 patients with PD were selected. According to the inclusion and exclusion criteria, 44 diabetics and 44 non-diabetics were enrolled in the two groups.

Patients were visited by two movement disorders fellowships and diagnosed with PD according to UK bank criteria (17). The inclusion criteria of our study included patients aged between $40-85$ years old with $P D$, who were referred to the Neurology Clinic of Rasoul Akram Hospital in 2019; all participants signed informed consent.

Patients with other diseases except PD, who manifest dyskinesia, were excluded from the study. Patients with PD (diagnosed with diabetes) were considered as the case group, while the control group consisted of non-diabetic patients with PD. Patients were assessed by a neurologist regarding demographic parameters, duration of diabetes and PD, dosage of levodopa, and presence of dyskinesia, its location, and time pattern.

Levodopa dosage, age, sex and PD duration were confounding factors, which may affect the desired causal correlation between diabetes and the presence of dyskinesia. The mentioned factors were equal as the baseline parameters between the two study groups. Data were subsequently analyzed using SPSS 22 (SPSS Inc., Chicago, Ill., USA).

\section{Ethics}

In this study, ethical protocols were followed according to the Declaration of Helsinki. The research was approved by the Ethics Committee of Iran University of Medical Sciences

(IUMS; IR.IUMS.FMD.REC1396.9021216146). All patients were informed about the aim of the study and asked to complete the informed consent form. All patients were also explained that their personal information will not be disclosed; every case was given a specific code number.

We confirm that we have read the Journal's position on issues involved in ethical publication and affirm that this work is consistent with those guidelines.

\section{Analysis}

Analysis in this study was performed using SPSS 22 (SPSS Inc., Chicago, Ill., USA). Descriptive statistics of quantitative variables were described by mean and variance; the qualitative data were defined by frequency $(17,18)$. Parametric or their equivalent non-parametric tests were used for analysis (19). A P-value=0.05 (95\% CI) was considered statistically significant (20).

\section{Results}

In this survey, 88 patients were assessed in two groups of diabetic and non-diabetic patients with PD; each group consisted of 44 patients. Patients were enrolled in this study after being confirmed regarding equality in their baseline characteristics. Descriptive statistics of demographic and clinical parameters of patients are illustrated in Table 1 and Figures 1 and 2.

Using the chi-square test, the prevalence of dyskinesia was higher (statistically significant) in diabetics than in non-diabetics $(P=0.033)$; the main hypothesis of this study was confirmed (Table 2).

All patients in the two study groups had similar baseline parameters, including levodopa dosage, sex category, and duration of PD involvement. All patients in the study groups were equal regarding the frequency of sex categories $(P=0.546)$. The analysis showed no significant difference in levodopa dosages between diabetics and non-diabetics groups $(P=0.703$; see Table 1$)$. PD duration was not significantly different between diabetic and nondiabetic patients $(P=0.148)$. The mean PD duration of diabetic and non-diabetic patients was 6.11 and 7.95 years, respectively (Table 1).

The frequency of dyskinesia location and time patterns are also shown in Figures 1 and 2. Simultaneous involvement of upper and lower extremities was the most prevalent location pattern of dyskinesia. Dyskinesia occurrence immediately after taking drugs was the most common time pattern of dyskinesia in diabetic patients with PD. 
Table 1. Descriptive statistics of qualitative and quantitative variables

\begin{tabular}{|c|c|c|c|c|}
\hline Variable & \multicolumn{2}{|c|}{ Category } & Mean \pm SD & (P-value) $^{\mathrm{a}}$ \\
\hline \multirow{3}{*}{ Age } & \multicolumn{2}{|c|}{ Diabetics } & $65.09 \pm 7.42$ & \multirow{2}{*}{$0.064^{\mathrm{b}}$} \\
\hline & \multicolumn{2}{|c|}{ Non-diabetics } & $61.59 \pm 9.85$ & \\
\hline & \multicolumn{2}{|c|}{ All cases } & $63.34 \pm 8.85$ & N/A \\
\hline \multirow{6}{*}{$\operatorname{Sex}$} & \multirow{2}{*}{ Diabetics } & Female & $45.5 \%$ & \multirow{4}{*}{$0.546^{c}$} \\
\hline & & Male & $54.5 \%$ & \\
\hline & \multirow{2}{*}{ Non-diabetics } & Female & $36.4 \%$ & \\
\hline & & Male & $63.6 \%$ & \\
\hline & \multirow{2}{*}{ All cases } & Female & $54.5 \%$ & \multirow{2}{*}{$\mathrm{N} / \mathrm{A}$} \\
\hline & & Male & $45.5 \%$ & \\
\hline \multirow{3}{*}{ Parkinson Duration } & \multicolumn{2}{|c|}{ Diabetics } & $6.11 \pm 3.49$ & \multirow{2}{*}{$0.148^{\mathrm{d}}$} \\
\hline & \multicolumn{2}{|c|}{ Non-diabetics } & $7.95 \pm 5.05$ & \\
\hline & \multicolumn{2}{|c|}{ All cases } & $7.03 \pm 4.41$ & N/A \\
\hline Diabetes duration & \multicolumn{2}{|c|}{ Diabetics } & $9.04 \pm 5.54$ & $\mathrm{~N} / \mathrm{A}$ \\
\hline \multirow{3}{*}{ levodopa dosage } & \multicolumn{2}{|c|}{ Diabetics } & $960.11 \pm 358.61$ & \multirow{3}{*}{$0.703^{\mathrm{d}}$} \\
\hline & \multicolumn{2}{|c|}{ Non-diabetics } & $1074.26 \pm 572.44$ & \\
\hline & \multicolumn{2}{|c|}{ All cases } & $1017.19 \pm 478.35$ & \\
\hline
\end{tabular}

A: Comparison between groups of diabetic and non-diabetic Parkinsonian patients; $b$ : T-test; $c$ : chi-square; d: Mann-Whitney; N/A: not applicable.

Table 2. Dyskinesia presence

\begin{tabular}{cccc}
\hline Category & Dyskinesia positive & Dyskinesia negative & P-value (Chi- square) \\
\hline All cases & $61.4 \%$ & $38.2 \%$ & \\
\hline Among diabetics & $86.4 \%$ & $13.6 \%$ & 0.033 \\
\hline Among non-diabetics & $36.4 \%$ & $63.6 \%$ & 0.00 \\
\hline
\end{tabular}

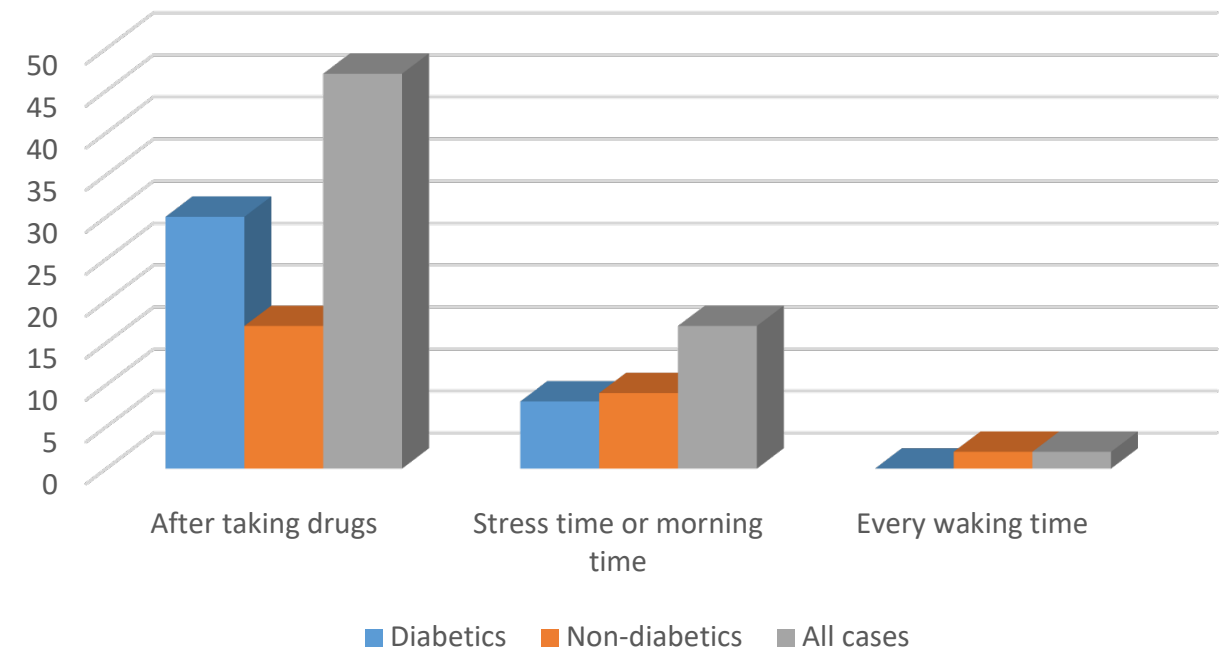

Figure 1. Frequencies of Dyskinesia time 


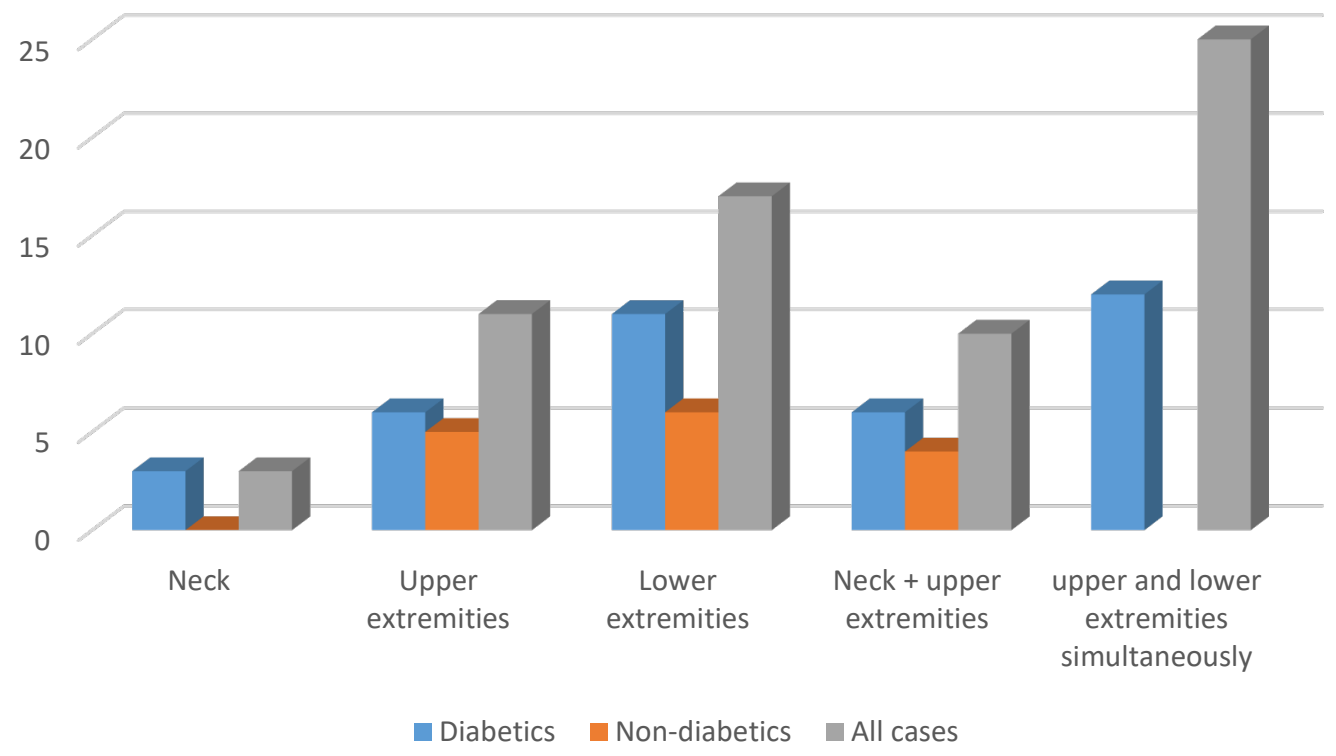

Figure 2. Frequencies of Dyskinesia location

\section{Discussion}

Based on our results, diabetic patients with PD had higher rates of dyskinesia compared with non-diabetic patients; it represented a higher risk of dyskinesia for diabetic patients who developed PD.

Levodopa compound medication is known as the main risk factor for dyskinesia development in patients with PD (8); the role of other risk factors of this debilitating complication has not been assessed yet, except in a few previous studies. The presence of DM not only increases the rate of PD (12) but also increases the risk of motor and postural disturbances in those patients (14) and leads to $36 \%$ aggravation of their clinical status (15).

As mentioned above, dopamine supplementation is a known risk factor for the incidence of dyskinesia. Hence, dyskinesia could occur in a prolonged course of PD and so the treatment course $(1,11)$. The diabetic subjects compared with non-diabetic ones were confirmed to be equal in baseline characteristics, including dopamine dosage and disease duration; equalization excludes the effect of mentioned confounding factors on the incidence of dyskinesia.

The pattern of dyskinesia in diabetic patients with PD regarding location showed the involvement of upper and lower extremities simultaneously. In terms of time, dyskinesia occurrence immediately after taking drugs was the most common pattern of dyskinesia in association with an increased risk by disease duration and higher levodopa dosage in those patients.

Regarding the equality of baseline characteristics, including age, disease and treatment duration, and levodopa compound medication dosage between diabetic and non-diabetic patients, as well as the incidence of LID during the natural course of treatment by levodopa compound medications, dyskinesia is more prevalent among diabetic patients; it indicates diabetes is an accelerating factor in the development of LID in PD.

Our results are consistent with the findings of previous studies; they indicated the increased risk of dyskinesia as a debilitating complication in patients with PD $(14,16)$. Tardive dyskinesia is also reported with a higher prevalence in diabetic subjects treated by neuroleptics, even in the absence of parkinsonism; the possible mechanism underlying its pathophysiology is attributable to dopamine receptor hyperfunctioning in hyperglycemic states (21-24). However, one researcher disagreed with the latter hypothesis (25).

It is highly recommended for future studies to assess the correlation between dyskinesia and the antidiabetic medication. Unfortunately, we did not assess the mentioned correlation, which is the limitation of this study. Furthermore, the role of the interval between levodopa regimen onset and LID onset has not been assessed or compared between diabetic and nondiabetic subjects, which should be addressed by future studies.

\section{Conclusion}

The comorbidity of DM and PD is a risk factor regarding the incidence of dyskinesia. Simultaneous involvement of upper and lower extremities (right after taking drugs) was the most common pattern of dyskinesia in diabetic patients with PD. 


\section{Acknowledgments}

We would appreciate Rasoul Akram hospital's attending, residents and nurses for their non-stop efforts for improvement of patients and for all their supports.

\section{Conflict of Interest}

The authors indicate that they have no financial or non-financial conflicts of interest or involvement with any organization in the subject matter or materials discussed in this manuscript.

\section{Funding source}

This study was funded by a research grant from Vice Chancellor for Research \& Technology of Iran University of Medical Sciences, Tehran-Iran. This research was approved by the ethics in the research committee of the Iran University of Medical Sciences (IUMS).

\section{References}

1. Taghizadeh G, Martinez-Martin P, Fereshtehnejad SM, Habibi SA, Nikbakht N, Alizadeh NH, Salehi S, Mehdizadeh M. Psychometric properties of the Berg balance scale in idiopathic Parkinson'disease in the drug off-phase. Neurological Sciences. 2018 Dec 1;39(12):2175-81. [DOI:10.1007/s10072-0183570-4]

2. Mehdizadeh M, Lajevardi L, Hassan Habibi SA,et al. The association between fear of falling and quality of life for balance impairments based on hip and ankle strategies in the drug on-and offphase of patients with idiopathic Parkinson'disease. Med J Islam Repub Iran. 2016. 30: 453.

3. Mehdizadeh M, Martinez-Martin P, Habibi SA, et al., The association of balance, fear of falling, and daily activities with drug phases and severity of disease in patients with Parkinson. Basic Clin Neurosci. $2019 . \quad 10(4)$ : 355. [DOI: 10.32598/bcn.9.10.295]

4. Marras C, Beck J , Bower J, et al. Prevalence of Parkinson's disease across North America. NPJ Parkinson's Disease. 2018; 4(1): 1-7. [DOI:10.1038/s41531-018-0058-0]

5. Levy G, Louis E, Mejia-Santana H, et al., Lack of familial aggregation of Parkinson disease and Alzheimer disease. Arch Neurol. 2004; 61(7): 1033-39. [DOI:10.1001/archneur.61.7.1033]

6. Sun Y, Chang Y, Chen H, Su Y, Fang Su H, Yi Li C. Risk of Parkinson disease onset in patients with diabetes: a 9-year population-based cohort study with age and sex stratifications. Diabetes $\begin{array}{llll}\text { Care. } & 2012 . & 35(5): & 1047-49 .\end{array}$ [DOI:10.2337/dc11-1511]

7. Zhu F, Li C, Gong J, Zhu W, Gu L, Li N. The risk of Parkinson's disease in inflammatory bowel disease: A systematic review and meta-analysis. Dig Liver Dis. 2019. 51(1): 38-42. [DOI:10.1016/j.dld.2018.09.017]

8. Thanvi B, Lo N, Robinson T. Levodopa-induced dyskinesia in Parkinson's disease: clinical features, pathogenesis, prevention and treatment. Postgraduate Med J. 2007. 83(980): 384-88. [DOI: 10.1136/pgmj.2006.054759]

9. Tolosa E. Advances in the pharmacological management of Parkinson disease, in Neuropsychopharmacology. 2003, Springer. 6578. [DOI:10.1007/978-3-7091-6020-6 4]

10. Huh E, Choi J, Sim Y, Sook Oh M. An integrative approach to treat parkinson's disease: ukgansan complements L-Dopa ameliorating dopaminergic neuronal damage and L-Dopa-induced dyskinesia in mice. Front Aging Neurosci. 2019. 10: 431. [DOI:10.3389/fnagi.2018.00431]

11. Sandyk R. The relationship between diabetes mellitus and Parkinson's disease. Int J Neurosci, 1993. 69(1-4): 125-30. [DOI: 10.3109/00207459309003322]

12. Xu Q, Park Y, Huang X, et al. Diabetes and risk of Parkinson's disease. Diabetes Care. 2011; 34(4): 910-15. [DOI:10.2337/dc10-1922]

13. Yang Y, Hsieh T, Li C, et al., Increased risk of Parkinson disease with diabetes mellitus in a population-based study. Medicine. 2017. 96(3): e5921

\section{[DOI:10.1097/MD.0000000000005921]}

14. Yue X, Li H, Yan H, Zhang P, Chang L, Li T. Risk of Parkinson disease in diabetes mellitus: an updated meta-analysis of population-based cohort studies. Medicine. 2016; 95(18): e3549 [DOI: 10.1097/MD.0000000000003549]

15. Schernhammer E, Hansen J, Rugbjerg K, Wermuth L, Ritz B. Diabetes and the risk of developing Parkinson's disease in Denmark. Diabetes care. 2011; 34(5): 1102-08. [DOI:10.2337/dc10-1333]

16. Kotagal V, Albin R, Müller M, Koeppe R, Frey $\mathrm{K}$, Bohnen N. Diabetes is associated with postural instability and gait difficulty in Parkinson disease. Parkinsonism Relat Disorder. $2013 . \quad$ 19(5): 522-26. [DOI:10.1016/j.parkreldis.2013.01.016]

17. Taghizadeh G, Martinez-Martin P, Meimandi M, et al. Barthel index and modified rankin scale: 
psychometric properties during medication phases in idiopathic Parkinson disease. Ann Phys Rehabil Med. 2019. [DOI:10.1016/j.rehab.2019.08.006]

18. Mehdizadeh M, Martinez-Martin P, Habibi SA, Fereshtehnejad SM, Abasi A, Niazi Khatoon J, Saneii SH, Taghizadeh G. Reliability and validity of Fall Efficacy Scale-International in people with Parkinson's disease during on-and off-drug phases. Parkinson's Disease. 2019 [DOI: 10.1155/2019/6505232]

19. Mehdizadeh M, Fereshtehnejad SM, Goudarzi S, et al., Validity and reliability of short-form McGill pain questionnaire-2 (SF-MPQ-2) in Iranian people with Parkinson's disease. Parkinsons Dis. 2020. 2020: 2793945 [DOI:10.1155/2020/2793945]

20. Mehdizadeh M, Mehraban A, Zahediyannasab R. The effect of group-based occupational therapy on performance and satisfaction of stroke survivors: pilot trail, neuro-occupational view. Basic Clin Neurosci. 2017. 8(1): 69. [DOI:10.15412/].BCN.03080109]

21. Caligiuri M.P, Jeste D. Association of diabetes with dyskinesia in older psychosis patients. Psychopharmacol. 2004. 176(3-4): 281-86. [DOI: 10.1007/s00213-004-1893-8]
22. Lévy E, Margolese H, Annable L, Chouinard G. Diabetes, tardive dyskinesia, parkinsonism, and akathisia in schizophrenia: a retrospective study applying 1998 diabetes health care guidelines to antipsychotic use. Can J Psychiatry. 2004. 49(6): 398-402. [DOI:10.1177/070674370404900611]

23. Ganzini L, Casey D, Hoffman W, Heintz R. Tardive dyskinesia and diabetes mellitus. Psychopharmacol Bull. 1992. 28(3): 281-86.

24. Lieberman JA, Alvir J. Diabetes and development of tardive dyskinesia. Am J Psychiatr. 1993. 1(50): 967.

25. Raja M, Azzoni A. Diabetes is not a risk factor for tardive dyskinesia: a retrospective observational study. Human Psychopharmacol. 2002. 17(1): 61-63. [DOI:10.1002/hup.354]

\section{How to Cite This Article:}

Mirzae M, Abbasi M H, Shahidi G A, Hosseinifakhr Q, Sina F, Habibi S A. Diabetes Increases the Incidence of Levodopa-Induced Dyskinesia in Parkinson's Disease; A Case-Control Study. J Adv Med Biomed Res. 2021; 29 (136) :258-263

\section{Download citation:}

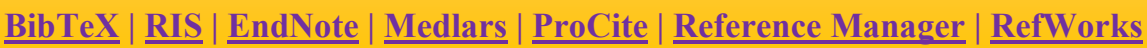

\section{Send citation to:}
(3). Mendeley
$\underline{\text { Zotero }}$ RefWorks RefWorks 